\title{
Phase differences in local field potentials from macaque monkey area V4 predict attentional state in single trials with $\mathbf{9 9 . 6 \%}$ accuracy David Rotermund*1, Simon D Neitzel ${ }^{2}$, Udo A Ernst ${ }^{1}$, Sunita Mandon ${ }^{2}$, Katja Taylor ${ }^{2}$, Klaus R Pawelzik ${ }^{1}$ and Andreas K Kreiter ${ }^{2}$
}

\author{
Address: ${ }^{1}$ Institute for Theoretical Physics, University of Bremen, Bremen, 28334, Germany and ${ }^{2}$ Institute for Brain Research, University of Bremen, \\ Bremen, 28334, Germany \\ Email: David Rotermund* - davrot@neuro.uni-bremen.de \\ * Corresponding author
}

from Eighteenth Annual Computational Neuroscience Meeting: CNS*2009

Berlin, Germany. 18-23 July 2009

Published: I 3 July 2009

BMC Neuroscience 2009, I0(Suppl I):P230 doi:10.1186/I47I-2202-I0-SI-P230

This abstract is available from: http://www.biomedcentral.com//47/-2202//0/SI/P230

(C) 2009 Rotermund et al; licensee BioMed Central Ltd.

Coherent oscillations and synchronous activity are suggested to play an important role in selective processing and dynamic routing of information across the primary visual cortical areas. In this contribution we show that phase coherency between distant recording sites allows to distinguish almost perfectly between two attentional states in a behavioral task, thus giving strong quantitative support for a functional role of oscillatory neural dynamics.

A macaque monkey was trained to perform a delayedmatch-to-sample task, in which the animal had to direct attention to one of two sequences of morphing shapes (1 deg. diameter) presented on a computer screen and separated by a gap of less than one degree of visual angle. The task was to signal the re-occurrence of the initial shape of the attended morphing sequence. Recordings of local field potentials (LFPs) were performed with an array of chronically implanted intracortical microelectrodes, covering parts of areas V1 and V4.

The LFPs were split into their frequency components by applying a Morlet-wavelet transform. From the transformed signals, we computed the phase coherency (a complex-valued scalar with amplitude $\leq 1$ and a phase) averaged over a time interval of $2500 \mathrm{~ms}$, for every electrode pair. We then used a support vector machine (SVM) to classify the attended state (attention directed either to one or to the other sequence) from the phase differences. Strikingly, nearly perfect state identification (up to $99.6 \%$ correct) was possible from several pairs of electrodes in $\mathrm{V} 4$, mainly in the frequency bands of $48 \mathrm{~Hz}$ and $61 \mathrm{~Hz}$. From V1-V4 electrode pairs, classification with up to $76 \%$ correct was possible.

Our results show that phase differences between signals from V4 can accurately inform about the direction of attention to different locations in visual space in every single trial. This effect is both, stable over time and robust against continuous changes of the shapes at the attended location. Our results give strong quantitative support for the hypothesis that coherent oscillations are a key mechanism underlying information processing under attention.

\section{Acknowledgements}

Support by BMBF Bernstein Group Bremen, DIP Metacomp, and the ZKW Bremen 